\section{EL CUERPO CONCEBIDO POR EL JUDO KODOKAN: UN PROYECTO EDUCATIVO DE PROGRESO TRUNCADO POR LA REACCIÓN POLÍTICA}

\author{
O CORPO CONCEBIDO PELO JUDÔ KODOKAN: UM PROJETO EDUCATIVO \\ DE PROGRESSO TRUNCADO PELA REAÇÃO POLÍTICA
}

THE BODY SEEN BY KODOKAN JUDO: AN EDUCATIONAL PROJECT OF PROGRESS HAMPERED BY POLITICAL REACTION

\section{Julián Espartero*}

Palabras clave Artes marciales. Cuerpo humano. Tabu.

Poder publico. Japón.

\section{Palavras-chave} Artes marciais. Corpo humano. Tabu.

Poder público. Japão.

\begin{abstract}
Resumen: El objetivo de este trabajo es exponer cómo el judo Kodokan -una genuina propuesta educativa que concibe el cuerpo como cauce de formación integral y de desarrollo humano y social-, fue sometido en Japón a una reaccionaria instrumentalización política que determinó su relegación por la versión propuesta por el ultranacionalismo. La metodología utilizada a lo largo del texto ha sido la propia de la investigación histórica: recolección, evaluación, verificación y síntesis de evidencias que han permitido obtener conclusiones válidas. Las cuales reflejan que, finalmente, fue la versión desnaturalizada del judo la que se impuso y se "exportó" a Occidente, soslayando la propia esencia de la concepción del judo de Kano y provocando que ésta todavía sea, prácticamente, desconocida por la mayoría de sus practicantes.
\end{abstract}

Resumo: $\mathrm{O}$ objetivo do presente trabalho é demonstrar como o judô Kodokan - uma proposta educativa que concebe o corpo como um instrumento para formação integral e desenvolvimento humano e social - foi submetido no Japão a uma instrumentalização política reacionária que determinou sua substituição por uma versão proposta pelo ultranacionalismo. A metodologia utilizada ao longo do texto foi a própria da pesquisa histórica: coleta, avaliação, verificação e síntese das evidências que permitiram obter conclusões válidas. Estas indicam que, finalmente, foi a versão desnaturalizada do judô que foi imposta e "exportada" ao ocidente, distorcendo a própria essência da concepção do judô de Kano e provocando que esta última ainda seja, praticamente, desconhecida pela maioria de seus praticantes.

Abstract: The aim of this work is to explain how Kodokan Judo - a genuine educational proposal that sees the body as a way for whole education and human and social development - was subjected to backwards political instrumentalization in Japan, causing its replacement by the version proposed by ultranationalism. The methodology used throughout this work has been that of historical research: collection, assessment, verification, and synthesis of evidence that has allowed us to reach valid conclusions. They point out that such denatured version of judo eventually prevailed and was 'exported' to the West, suppressing the very nature of the conception of Kano judo and causing it to be virtually unknown to most practitioners.
*Universidad de León. León. España. E-mail: jespc@unileon.es

Recebido em: 05-03-2016 Aprovado em: 22-09-2016 (c) (i) (8) Licence 


\section{INTRODUCCIÓN}

El periodo denominado Restauración Meiji (1868-1912) marca la restitución del poder militar y político deł Japón a la autoridad imperial, determinando decisivamente el inicio de un proceso de modernización y transformación de su estructura política feudal. Lo cual resultaba ser preciso para convertirse en una gran nación y ocupar lugar en el plano internacional. $A$ tal fin, las élites políticas consideraron necesario reforzar la unidad del Estado y una población leal, obediente, dispuesta a realizar todo tipo de sacrificios por el bien de la colectividad nacional.

Para lograrlo, se promovió una ideología nacionalista basada en el concepto de un gran Estado-familia -cuya razón última era el culto al Emperador, como dios y padre de todos los japoneses- y en la construcción de una identidad nacional que representara al Japón moderno pero, también y sobre todo, que fuera expresión de la genuina esencia de los valores únicos y eternos de la nación japonesa, que la hacían no sólo distinta del resto de los países, sino también superior a ellos. Así, se produjo una profunda remoción de la sociedad, pero ésta, lejos de afectar a la plena vigencia de virtudes tradicionales, reforzó el sentimiento de preservarlas y legitimó un temprano nacionalismo de carácter espiritual e imperial construido sobre el concepto Kokutai que, de alguna manera, puede traducirse como identidad o esencia nacional japonesa (LAVELLE, 1990; SHIMAZU, 2006).

La misma encontró su más ilustrativa expresión en el Bushido - el código no escrito de conducta del samurai-, que sirvió de cauce ideal para la configuración de esa nueva identidad nacional, mediante la transposición artificial de aquel supuesto y exclusivo sistema ético a la totalidad de la nación con un doble objetivo: regular la moral de la vida social y ser una herramienta para la creación de la moderna identidad nacional japonesa (NARROWAY, 2008; BENESCH, 2011). En esta contextualización la política nacionalista vio en el bujutsu ${ }^{1}$ un medio de difusión del Bushido que podía ser muy adecuado para unificar el espíritu, los pensamientos y las acciones de los ciudadanos en un cuerpo político nacional que sustentara al nuevo Estado pretendido (WARNER; DRAEGER, 1982; PATTERSON, 2008; DE CRÉE, 2013; GAINTY, 2013).

Según Inoue (1998), es por esto que el bujutsu fue sustituido por el budo², una "invención moderna" originalmente concebida como una forma cultural híbrida, generada para la modernización de la práctica tradicional e ilustrativa del fenómeno que Hobsbawm (2002) designara como "invención de la tradición". Señalando al judo Kodokan, fundado por Kano, como "el primer ejemplo de la invención del budo" (INOUE, 1998, p. 163). Sin embargo, en la presente exposición postulamos que esta categorización del judo Kodokan como "tradición inventada" pueda ser replanteada en los términos señalados por Cohen (1982), cuando afirma que la tradición tiene valores pragmáticos, en cuanto que pueden adecuarse o responder a los requerimientos de nuevas situaciones. De ahí que "Los individuos pueden destacar las raíces históricas de algunas prácticas, no con el fin de demostrar su antigüedad, sino para exhibir su adecuación y viabilidad a ciertas circunstancias sociales" (COHEN, 1982, p. 5).

Como se pondrá de manifiesto, el judo Kodokan fue un método de educación integral basado en la adecuación de una práctica corporal tradicional marcial a la mejora y

1 Dicho término designaba las técnicas y métodos de combate desarrollados y practicados principalmente por los miembros de la clase militar (bushi o samurai). Dada la influencia de los mismos, dicha designación identificaba semánticamente a la generalidad de las artes marciales de Japón. 
perfeccionamiento de los individuos y, por ende, de la vida social. En su manejo de la tradición, Kano no inventó ni buscó un continuismo artificial con el pasado sino que procuró su adaptación, mezclándola con la modernidad, para obtener una formación que respondiera a una exigencia de progreso: la susodicha mejora individual y social.

A pesar de que esta firme vocación educativa le llevó a sostener una independencia que preservara el judo de la instrumentalización pretendida por el ultranacionalismo (STEVENS, 1995; PATTERSON, 2008, DE CRÉE, 2013), los avatares sociopolíticos del momento fueron minando dicho empeño y su proyecto de progreso finalmente fracasará, hasta el punto de que su método educativo fue completamente desnaturalizado al ser fagocitado para servir a los intereses del poder político.

La razón ultranacionalista, pues, influyó decisivamente en el judo desde sus inicios y fue progresivamente trastocando su finalidad original hasta terminar desfigurándola. Sin embargo, y como bien señala Brousse (2011), la mayoría de los estudios realizados al respecto han ignorado esta instrumentalización política y el efecto que la misma deparó. De ahí que el presente trabajo pretenda, también, poner de manifiesto el rigor de esta intervención y la relevancia de sus consecuencias.

\section{EDUCACIÓN Y POLÍTICA DEL CUERPO EN EL JAPÓN MEIJI}

La política de Meiji consideró a la educación como la clave principal para la formación de una mentalidad colectiva que permitiera al país alcanzar sus objetivos, procediendo a la absorción del conocimiento occidental. Sin embargo, debe precisarse que esta importación cultural se llevó a cabo de forma selectiva por los dirigentes políticos que, como bien explica Craig (1995), la tamizaron a través del filtro de las tradiciones japonesas para reafirmar las fuentes de su identidad nacional (Kokutal). De forma que esta particular forma de asimilación cultural fue designada mediante la expresión popular wakon yo sai (técnica occidental y espíritu japonés) (ABE, 1986).

Esta situación dio origen a una profunda tensión cultural que, según Nagai (1969), resultó ser el lastre más oneroso de la educación Meiji, pues hubo de enfrentarse a la difícil tarea de manejar simultáneamente los dos polos culturales de la misma: modernidad y tradición. Lo cual ya tuvo su reflejo en la primera Ordenanza de Educación que impuso, en 1872, un sistema de educación obligatoria (Gakusei), si bien con la finalidad primordial de perseguir la prosperidad del Estado y, en todo caso, la inculcación de una lealtad absoluta al Emperador. De manera que esta implementación educativa proporcionó al Estado un total acceso a las jóvenes generaciones, que le permitió transmitir los valores fundamentales de la ideología oficial y, con ello, el desarrollo de los sentimientos de identidad nacional, lealtad y sumisión al Emperador (MANZENREITER, 2007).

Pronto se detectó, sin embargo, que el sistema educativo adoptado no terminaba de encajar bien con la sociedad japonesa que se pretendía, entendiéndose que ello debía de corregirse a través de la introducción en el sistema de enseñanza de la educación moral (shushin) que integraba una compleja mezcla de ideales confucianos básicos y de sentimientos imperiales y nacionalistas (IKEMOTO, 1996). Por tanto, con la Orden de Educación de 1879 y su modificación de 1880, se inició su implantación, que se irá consolidando para culminar con el triunfo de los argumentos conservadores relativos a la necesidad de una educación moral acorde 
con los tradicionales valores japoneses y diferenciada de la moralidad de los europeos. Esto es lo que representó la promulgación, en 1890, del Edicto Imperial sobre la Educación (Kyoiku Chokugo). Con ello, la educación y la cultura en general se tornaron en sistemas politizados, implicando la ruptura del Japón Meiji con el núcleo de la tradición occidental. Este momento fue clave, a partir de aquí el ideal educativo se colocó al servicio del Estado y será el germen del ultranacionalismo militarista que se desarrolló en las décadas posteriores (NIEVES, 2014).

Esta exhaustiva politización no dudó en instrumentalizar y aprovechar la expresión simbólica de un cuerpo superior, fuerte y lleno de salud para entrañar el sentimiento de pertenencia a la nación y para formar el imaginario, los ritos y la retórica de la misma. De modo que, en el marco de la educación obligatoria, la Educación Física (taiso) "[...] asumió el papel de formar el cuerpo y la mente de los alumnos para servir al Estado-Emperador" (KURISU, 2000). En esta contextualización, y como se ha anticipado, surgió la consideración de que el bujutsu pudiera ser muy adecuado para los fines pretendidos. Pero debe precisarse que, en el ambiente de cambios y transformaciones generalizado en la sociedad japonesa del momento, la estima del bujutsu había decaído notablemente como consecuencia de su identificación con la imagen del Japón feudal que generaba un gran rechazo popular. Este motivo fue lo que determinó su sustitución por el budo, que fue ideado por el ultranacionalismo como un elemento que contrarrestara los valores occidentales e infundiera «la cultura del deporte moderno de Japón con "espíritu japonés" (INOUE, 1998, p. 164). En suma, el budo fue visto como un medio de preservar el Bushido, identificándolo como un sistema de valores tradicionales japoneses y que finalmente será utilizado como un medio de inculcar la aludida educación moral (shushin).

Sin embargo, esto no se producirá de forma inmediata y necesitará un proceso que se verá inmerso en tensiones, propias del momento político, entre los dirigentes favorables a las metodologías educativas occidentales y los conservadores nacionalistas que buscaban imponer estos valores tradicionales en el sistema educativo japonés. En cualquier caso, en este periodo se inicia la imbricación de una Educación Física obligatoria en la que el cuerpo y su educación sean instrumento de las políticas dirigidas al desarrollo de los sentimientos de identidad nacional, lealtad y sumisión. En tal sentido, la Ordenanza de 1872 determinó que, en el nivel de Primaria y en esta materia, la formación consistiera esencialmente en juegos y en ejercicios de calistenia que se practicaban de acuerdo a las órdenes militares proporcionando así técnicas especialmente adecuadas para desarrollar actitudes de subordinación colectiva (KURISU, 2000). Asimismo, desde la década de 1880 y con idéntica finalidad, los ciclos superiores incluyeron ejercicios de gimnasia militar (heishiki taiso) que se practicaba con equipo militar, fusiles de madera e integrando marchas y simulación de combates (MANZENREITER, 2007).

La primera mención oficial tendente a la incorporación del budo al sistema escolar se registró en 1883 -en respuesta a las presiones que pedían esta inclusión-, encargándose al Instituto Nacional de Gimnasia (Taiso Denshusho) que llevara a cabo un informe para determinar la viabilidad de la inclusión curricular del kendo y el jujutsu (UOZUMI, 2010). Dicho informe se concluyó en 1884 y en el mismo se desaconsejó la enseñanza escolar de dichas disciplinas, al considerarlas «inadecuadas como asignatura ordinaria» e indicando que su inclusión -fundamentada simplemente en el hecho de que constituían una tradición nacional y centrándose excesivamente en la educación moral- podía dar lugar a un desequilibrio en el planteamiento educativo que provocara que "[...] los genuinos beneficios de la educación física no fueran factibles" (NIEVES, 2014, p. 68). 
En 1886, fue nombrado ministro de educación Arinori Mori -un nacionalista convencido y, a la vez, un defensor de los métodos occidentales de educación-, quien consideraba la educación física como un excelente método de mejorar la salud e inculcar el patriotismo (GUTTMANN; THOMPSON, 2001). Para estos fines consideró especialmente indicada la gimnasia militar y trató de forjar el cuerpo y espíritu de la nación japonesa a través de la misma, excluyendo las prácticas corporales tradicionales que integraban el budo del sistema de enseñanza, al considerarlas un anacrónico método de educación. Por tanto, la gimnasia militar pasó a formar parte del currículo escolar a través de las Ordenanzas de la Universidad Imperial, la Escuela Normal, la Escuela Secundaria y de la Escuela Primaria, publicadas en 1886 (DUKE, 2008). A partir de ese momento, la gimnasia militar quedó establecida en todos los niveles del sistema educativo con la finalidad de formar cuerpos fuertes y obedientes con un patriotismo sin fisuras (ABE et al., 1992).

\section{EL JUDO KODOKAN: EL CUERPO COMO INSTRUMENTO DE DESARROLLO HUMANO Y SOCIAL}

Este contexto político-social es el que impera cuando Jigoro Kano crea oficialmente, en 1882, el judo Kodokan. Su brillante trayectoria como educador a través de los altos cargos que desempeñara y su inconmensurable labor para reformar y modernizar el sistema educativo del Japón, da buena cuenta del estrecho vínculo que guardara su método con la educación (DRAEGER, 1974; STEVENS, 1995, 2013; WATSON, 2000; MAZAC, 2006).

Durante su adolescencia y a pesar de su brillantez académica, Kano se vio relegado a una posición de subordinación debido a su inferioridad física. Ello le indujo a buscar un remedio que le permitiera solventar esta situación y, al ingresar en la universidad, comenzó la práctica del jujutsu ${ }^{3}$, pero no sólo por su eficacia como arte marcial sino también por apreciar el valor del mismo como una práctica corporal tradicional propia de la cultura nacional que se debía preservar (DRAEGER, 1974; STEVENS, 2013). Sin embargo, esto no impediría que llevara a cabo su reformulación: "[...] el estudio del jujutsu me llevó a formular un concepto completamente nuevo en el que la práctica tenía como objetivo la mejora física y mental, superando su sentido tradicional que era sólo la preparación para el combate" (KANO, 1998, p. 69).

Será, precisamente, este profundo y respetuoso conocimiento del jujutsu el que permitió a Kano "subvertir" su sentido tradicional. Pero, en todo caso y si se nos permite la expresión, se trató de una "subversión" evolutiva que aportaba mejora o progreso, en cuanto pretendía ser instrumento real de una formación integral en el plano individual y social. Para ello, primeramente, configuró el judo Kodokan "[...] reuniendo los aspectos positivos encontrados en todas las escuelas de jujutsu, revisados a la luz de los principios científicos" (KANO, $1889^{4}$ apud CADOT, 2013, p. 123). Esto supuso una innovadora aportación sustentada en el análisis científico de las técnicas -fundamentalmente desde la biomecánica y la fisiologíapara minimizar el riesgo lesivo y hacer más adecuada la práctica a la sociedad del momento (WATSON, 2000; MAZAC, 2006; CADOT, 2007; BARIOLI, 2010; GAINTY, 2013).

\footnotetext{
3 El jujutsu se configuraba como el arte o técnica (jutsu) de la adaptabilidad (ju). Es decir, el arte de adaptarse a la forma más adecuada para vencer al adversario y se integraba por una serie de técnicas de combate que utilizaban el cuerpo humano como arma a través de proyecciones, inmovilizaciones, estrangulaciones, luxaciones, golpeos, etc.

4 KANO, Jigoro, en su conferencia "Del judo y de su valor tanto educativo como pedagógico" (Judo-ippan-narabi-ni-sono-Kyoikujo-no-kachi) , pronunciada el 11 de mayo de 1889, ante la Asociación Pedagógica del Gran Japón (Dai-nihon-kyoiku-kai).
} 
Pero su iconoclasia fue más allá y se extendió a hacer de su método un sistema de enseñanza abierto y gratuito para todos aquellos que quisieran seguirlo, rompiendo el tradicional carácter retribuido y secreto de este tipo de enseñanzas, orientado no sólo a preservar la eficacia de las técnicas sino también su interés comercial (CADOT, 2013). Lo cual hallaba su justificación en que, para Kano, lo importante no es la adquisición del conocimiento de la técnica de combate, sino los efectos que produce su práctica sobre el individuo. Es esta circunstancia la que le lleva a interesarse por una "cultura del cuerpo" (INOUE, 1998) en la que la práctica corporal se realiza en ausencia de todo discurso o ejercicio específico para el espíritu, pues observa que la solicitación física del combate per se entraña efectos sobre las cualidades intelectuales y morales del practicante.

Por tanto, crea el judo Kodokan como un sistema de educación física que desarrolle un cuerpo sano y vigoroso que pueda actuar como cauce de la mente y del espíritu (SHIMIZU, 2008). Pues esta práctica corporal, a su vez, solicitará inteligencia, primeramente a través del trabajo de memorización, de imitación de lo que se ha visto; después, y ante todo, por la necesidad de encontrar la solución más adecuada en función de los medios de los que uno dispone (su cuerpo, su inteligencia, su nivel de experiencia). Lo que determinará la educación intelectual del judo (MAZAC, 2006). Asimismo, el enfrentamiento corporal que supone, llevará a que el practicante sienta de forma más vívida el problema que se le plantea, que lo experimente físicamente y que lo gestione con todo su ser, percibiendo plenamente lo que es comprometerse, lo que es fracasar, el valor de volver a buscar una solución, de no abandonar. Todo esto formará una actitud del espíritu, es un proceso de educación moral (CADOT, 2007).

Debe afirmarse, entonces, que el judo Kodokan busca a través de la práctica corporal la formación integral, entendiéndola como una transformación del individuo construida mediante un trabajo de uno mismo sobre sí mismo, que emana de un entrenamiento cotidiano y a menudo riguroso que progresivamente mejorará la condición física, animando una evolución positiva del estado intelectual y espiritual. Produciéndose así una suerte de espiral evolutiva donde cada uno de estos elementos es el motor de los otros (ESPARTERO et al., 2011). El cuerpo es, pues, el instrumento a través del cual se verifica aquella formación integral del practicante que, más allá de los fines del combate, le permite alcanzar su perfección personal (jiko no kansei). La cual resulta ser precisa para conseguir su última causa: "[...] el objetivo del judo es perfeccionarse para después contribuir a la sociedad" (KANO, 1998, p. 166).

En efecto, la práctica del judo Kodokan pretende conseguir el perfeccionamiento individual, pero con la consciencia de que esta propia perfección sólo puede ser alcanzada mediante la comprensión de la ayuda y prosperidad mutua (Jita Kyoel) que implica que cuando se alcanza la propia prosperidad es necesario ayudar a los otros a hacer lo mismo. Lo cual sólo será posible

[...] cuando uno está físicamente sano y en posesión de un alto nivel intelectual y moral [...]. A partir de aquí, uno debe esforzarse para obtener su propia perfección, así como para el desarrollo y la prosperidad de los demás. Para obtener el verdadero beneficio personal, se ha de tener también en consideración el beneficio de la sociedad, pues la mayor prosperidad de uno mismo ha de ser lograda a través del servicio a la humanidad [...] El último objeto del judo, es también el último objeto del hombre: la perfección de uno mismo para servir a la humanidad (KANO, 1998, p. 23). 
El cuerpo, así concebido por Kano, es el nexo fundamental de una clara interdependencia entre la mejora individual y el desarrollo del bienestar y progreso social (SHIMIZU, 2008). Como señala Cadot (2007), en esta concepción el cuerpo aparece como un vehículo. Primeramente, de uno mismo hacia sí mismo y, a continuación, de uno mismo hacia el otro, los otros. Finalmente el cuerpo "[...] podrá revelarse como un vehículo hacia una sociedad mejor, integrada por personas que se sienten bien consigo mismas y que tienen una buena relación con los demás" (CADOT, 2007, p. 85). De ahí que pueda decirse que Kano concibe el cuerpo como un medio multidimensional para la constitución de la sociedad, de modo que "[...] el cuerpo y la sociedad y su constitución mutua se funden a lo largo del tiempo en un vivo proceso corpóreo" (BROWN, 2005, p. 25).

En definitiva, Kano reivindica con su método la idoneidad de las prácticas corporales marciales - entendidas en el estricto sentido que les diera Mauss (1934, p. 1): "[...] las maneras en que los hombres, en cada sociedad, saben servirse de sus cuerpos de un modo tradicional"como instrumento de formación integral. Como una práctica, en fin, cuyo significado trasciende a la acción, dotando a la corporeidad de un sentido orientado hacia el desarrollo humano y social. Lo cual contrasta fuertemente con la instrumentalización del judo -y del conjunto del budo- que realizará el ultranacionalismo con la finalidad de construir una nueva identidad nacional japonesa, así como un dispositivo del saber/poder encaminado a crear "cuerpos sometidos y ejercitados" (FOUCAULT, 2000, p.160).

\section{EL EMPEÑO EDUCATIVO DE PROGRESO TRUNCADO: EL TRIUNFO DE LA REACCIÓN}

Si bien Kano nunca participó en la política activa, sí pretendió que el judo Kodokan contribuyera educativamente a la formación del nuevo Estado japonés (NAKAJIMA; THOMPSON, 2012). Con esta finalidad solicitó al Gobierno la inclusión del judo en el currículo escolar. El 11 de mayo de 1889, y debido a su incipiente prestigio en el ámbito de la educación, tuvo la oportunidad de llevar a cabo la primera presentación teórica de su método. Invitado por la influyente Asociación Pedagógica del Gran Japón (Dai-nihon-kyoiku-kai), impartirá la paradigmática conferencia "Del judo y de su valor tanto educativo como pedagógico" (Judoippan-narabi-ni-sono-Kyoikujo-no-kachi) ante el nuevo ministro de Educación, altos funcionarios del Ministerio, pedagogos y diplomáticos occidentales. En la misma presentó el judo Kodokan como un sistema innovador que, basándose en tradicionales prácticas corporales marciales, las engloba y las trasciende a través de la mejora individual y social que constituye su objetivo. Una formación global, en suma, que viniendo de la más antigua tradición se proyectaba hacia al futuro como una vía de progreso (MAZAC, 2006; NIEHAUS, 2006; CADOT, 2007, 2013; DE CRÉE, 2013).

Asimismo, rebatió los argumentos del negativo informe de 1884 y afirmó que la formación integral planteada no resultaba ser accesible a través de las "gimnasias occidentales" implantadas institucionalmente, al centrarse exclusivamente en la vertiente física de la educación. Finalmente, concluyó la idoneidad del judo como método educativo: "Si incluimos el judo como asignatura en el currículo escolar de nuestro país, ello sin duda puede colmar las lagunas del sistema educativo actual y afianzar la formación del carácter de nuestros alumnos" (KANO, 18895, apud CADOT, 2013, p. 182).

5 KANO, Jigoro, "Del judo y de su valor tanto educativo como pedagógico" (Judo-ippan-narabi-ni-sono-Kyoikujo-no-kachi), citada en la anterior nota al pie. 
En definitiva, en esta primera exposición pública ya puede verse el germen del postulado educativo que Kano sostendrá en las múltiples actividades, conferencias y escritos que realizó a lo largo de su vida. El objetivo primordial de su método, el servicio a la humanidad, la ayuda y prosperidad mutua (Jita Kyoei). Esto es, la divisa, el ideal de progreso del judo Kodokan.

Por lo demás, esta conferencia no consiguió su finalidad y las tensiones ideológicas del periodo retrasarán la inclusión escolar del judo casi tres décadas, a pesar de la perseverancia de Kano en su iniciativa educativa (NAKAJIMA; THOMPSON, 2012). Sin embargo, la misma irá zozobrando a medida que la voluntad política fuera decantándose por la inclusión escolar del budo con el afán, eminentemente instrumental, de configurarlo como un cauce que permitiera establecer vínculos inmediatos con la artificial continuidad histórica y tradicional del Bushido (INOUE, 1998; MANZENREITER, 2007; PATTERSON, 2008; ROBERTS, 2009; DE CRÉE, 2013).

Así, en 1894, la primera guerra chino-japonesa alentará un auge del ultranacionalismo que se tradujo en un renacimiento del "tradicional espíritu marcial" (STEVENS, 1995, p. 31). Esto propició que, en 1895 y con el patrocinio de la familia imperial, se fundara la Dai Nippon Butokukai ("Asociación de las virtudes marciales del gran Japón") con el objeto de revitalizar la práctica del budo "frente a la enervante modernidad occidental" (GAINTY, 2013, p. 16). La misma alcanzó pronto un rápido crecimiento y expansión que usurpará la influencia del judo Kodokan de Kano, convirtiéndose en la entidad institucional más importante al erigirse en "la gran responsable de la codificación y nacionalización de la práctica del budo, especialmente kendo y judo, [...] y por sus exitosos esfuerzos para incluirlas en los currículos de la escuela pública" (GAINTY, 2013, p. 35).

A partir de 1896 se plantearon nuevas iniciativas parlamentarias tendentes a la inclusión escolar del budo en el currículo, hasta que - en 1908 - la Dieta Imperial aprobó la integración de kendo y judo como asignaturas optativas en el mismo. Esta política instrumental irá calando en la sociedad y, ya en los años veinte, el budo en general experimentó un crecimiento notable que se manifestó particularmente en el judo (INOUE, 1998). Sin embargo, Kano mostró públicamente su insatisfacción con la deriva que el mismo estaba tomando -principal motivo de sus constantes tensiones con la Butokukai (GAINTY, 2013)-:

El judo Kodokan se ha extendido hasta contar con veinte mil miembros directos, mientras que el total de los que practican en diversas escuelas equivale a unos cuantos millones. [...] observo que [...] su progreso se ha limitado esencialmente a la dimensión física, sin ser capaz de desplegar el potencial cultural y espiritual que el judo puede aportar a la sociedad (KANO, 1998, p. 69).

La invasión japonesa de Manchuria, en 1931, originó un agudo repunte del ultranacionalismo militarista, que fue causa directa de que ese mismo año se modificara la Orden reguladora de la Escuela Secundaria para incluir el judo -juntamente con el kendocomo asignatura escolar obligatoria, al considerarse que ambas eran "artes marciales únicas que en nuestra nación nutren un espíritu nacional puro y robusto" (NIEVES, 2014, p. 71). Esto precipitará la disolución de la genuina finalidad educativa del judo Kodokan, en cuanto que recrudeció la reaccionaria instrumentalización política que venía padeciendo y vino a convertirlo en la 
máquina eficiente", siendo dicha máquina el propio Estado japonés (ROBERTS, 2009, p. 49).

Prevaliéndose de su prestigio e influencia, Kano hizo lo posible por mantener su judo Kodokan al margen de las corrientes militaristas, constituyendo "un ejemplo excepcional" (ABE et al., 1992, p. 9). Sin embargo, la apropiación política del judo era inevitable si se tiene en cuenta que la Butokukai se configuró institucionalmente como el centro nacional de la enseñanza del judo, a pesar de la oposición que Kano siempre manifestó: "Ha de objetarse ¿cómo nunca la Butokukai adoptó otro judo que el del Kodokan y sin embargo se ha convertido en su sede central?" (KANO, 1998, p. 104). Es más, como relatan De Crée y Jones (2011, p. 120), hacia el final de su vida, Kano era ya perfectamente consciente de que "el modelo pedagógico que se había propuesto para el judo era un objetivo fallido".

Tras el fallecimiento de Kano se culminó definitivamente esta apropiación por el Estado, alcanzando su cenit con la absoluta militarización que experimentará el budo por la entrada en la Segunda Guerra Mundial y su sometimiento al esfuerzo bélico que suponía. A este fin, en 1942, se reorganizó la Butokukai que pasó de depender directamente de la Casa Imperial a ser una entidad gubernamental dependiente del Ministerio de Salud y trasladándose su sede a Tokio. A su vez el Kodokan perdió su identidad y todo atisbo de independencia al ser integrado en la estructura de la Butokukai y quedar sometido a su supervisión. La finalidad de esta medida no podía ser más explícita:

Los súbditos del Imperio japonés deben estudiar budo para cultivar la lealtad, la valentía y el heroísmo con el fin de reforzar el espíritu de la nación (...). La esencia del budo debe encarnar el espíritu de la nación, y en caso de peligro, no se debe vacilar en sacrificar la vida cumpliendo la obligación debida al Emperador (BENNETT, 2014, p. 74).

Así pues, el ideal del Bushidose convirtió en la última causa del judo. El ultranacionalismo militarista, la reacción, había triunfado.

\section{CONCLUSIÓN}

Durante la guerra, las actividades del Kodokan prácticamente se paralizaron y, al finalizar la contienda, las fuerzas de ocupación aliadas prohibieron la práctica del budo, incluyendo al judo. Asimismo, la exacerbada instrumentalización política a que fue sometido tuvo como consecuencia principal que el modelo educativo propuesto por Kano fuera relegado por la "propuesta oficial" que fue la predominante en Japón (GLEESON, 1984). Ello implicó que se enseñaran las bases técnicas del judo Kodokan, pero distorsionando su finalidad de formación global. De modo que, como afirma Brousse (2005), fue esta especie de versión del jujutsu reexaminada por el ultranacionalismo y no el método de Kano lo que sobrevivió y lo que se "exportó" a Occidente. Lo que ha provocado que la propia esencia de la concepción del judo todavía sea, prácticamente, desconocida por la mayoría de sus practicantes (DE CRÉE, 2013).

Esto supone tanto como ignorar que la propuesta educativa de Kano superó el sentido tradicional de una práctica corporal marcial, adecuándola a un afán humanista de progreso. Aunque ello supusiera contrariar la interpretación oficial de la tradición como un valor inmutable impuesto por el pasado, para reivindicar la misma como un valor susceptible de ser adecuado al continuo avanzar del tiempo y contribuir así a que el mismo supusiera progreso. 
Así, el judo Kodokan, el cuerpo concebido por Kano, parte a la conquista del progreso a través de una práctica corporal que pretende que el perfeccionamiento personal construido por uno mismo sobre sí mismo, deba ser puesto al servicio de la sociedad, de la humanidad. A su vez esta cooperación y prosperidad mutua permitirá acrecentar al máximo el desarrollo personal, para que cada individuo sea lo mejor posible en adecuación a su mismidad, a sus aspiraciones y a su potencial.

Hay razones, pues, para insistir en la difusión y reivindicación de estos presupuestos. Para tratar de hacer que el judo se convierta en aquello que no llegó a ser: el cuerpo como instrumento de progreso y humanismo. Creemos, con Barioli (1998, p. 15), que este propósito, asumido en una época difícil, sigue manteniendo una extraordinaria vigencia, pues refiere "[...] a una educación que trasciende los ideales del pasado, a fin de que cada generación deje un mundo mejor que el que encontró."

\section{REFERENCIAS}

ABE, Shinobu. Zen and Sport. Journal of the Philosophy of Sport, v. 13, n.1, p. 45-48, 1986. ABE, Ikuo; KIYOHARA, Yasuharu; NAKAJIMA, Ken. Fascism, sport and society in Japan. The International Journal of the History of Sport, v. 9, n. 1, p. 1-28, 1992.

BARIOLI, C. Prefazione. In: KANO, J. Fondamenti del Judo. Milano: Luni Editrice, 1998. p. 9-17.

BARIOLI, Cesare. Kano Jigoro Educatore: il vero Judô. Milano: Nuove Operazioni Editoriali, 2010.

BENNETT, Alexander. A Reconsideration of the Dai-Nippon Butokukai in the Purge of Ultranationalism and Militarism in Post-war Japan. 2014. Disponible en: <https://kiss.kokushikan.ac.jp/ pages/contents/0/data/1005290/0000/registFile/1346 194x 029 06.pdf>. Acceso en: 27 sep. 2016.

BENESCH, Oleg. Bushido: the creation of a martial ethic in late Meiji Japan. Tesis (Doctoral). 2011. University of British Columbia, Vancouver, 2011.

BROUSSE, Michel. Les racines du judo français. Histoire d'une culture sportive. Bordeaux: Presses Universitaires de Bordeaux, 2005.

BROUSSE, Michel. Ondes de choc; conflits politico-culturels et évolution du judo mondial. In: TERRET, T. Histoire du sport et géopolitique. Paris: L' Harmattan, 2011. p. 55-75.

BROWN, David. Cuerpo, cultura y transmisión en la actividad física: considerando el ejemplo de las artes marciales tradicionales. In: BORES N. J. La formación de los educadores de las actividades físico-deportivas extraescolares. Palencia: Cuadernos Técnicos, 2005. p. 23-35.

CADOT, Yves. Kanô Jigorô et le corps véhicule. In: BROTONS, A.; GALAN, C. Japon Pluriel 7. Actes du septième colloque de la Société française des études japonaises, Arles: Éditions Picquier, 2007. p. 81-90.

CADOT, Yves. Du judo et de sa valeur éducative comme pédagogique. France: Metatext, 2013.

COHEN, Anthony P. Belonging: the Experience of Cultura. In: COHEN, Anthony P., Belonging, identity and social organization in British rural cultures. Manchester: Manchester University, 1982. p. 1-17.

CRAIG, Darrell Max. Japan's Ultimate Martial Art: Jujitsu Before 1882. Rutland:Tokyo: Tuttle, 1995. 
DE CRÉE, Carl. Shōnen Jūdō-no-kata [,Forms of Jūdō for Juveniles']: an experimental Japanese teaching approach to jūdō skill acquisition in children considered from a historic-pedagogical perspective - part 1. Journal of Combat Sports and Martial Arts, v. 4, n. 1, p. 1-13, 2013.

DE CRÉE, Carl; JONES, Llyr C. Kōdōkan Jūdō’s Inauspicious Ninth Kata: The Joshi goshinhō - "Selfdefense methods for females" - Part 1. Archives of Budo, v. 7, n. 3, p. 105-123, 2011.

DRAEGER, Don F. The Martial Arts and Ways of Japan: Modern Bujutsu \& Budo. New York: Weatherhill, 1974. v.3.

DUKE, Benjamin. The History of Modern Japanese Education: Constructing the National School System, 1872-1890. New Brunswick: Rutgers University, 2008.

ESPARTERO, Julián; VILLAMÓN, Miguel; GONZÁLEZ, René. Artes marciales japonesas: prácticas corporales representativas de su identidad cultural. Movimento, v. 17, n. 3, p. 39-55, jul./set. 2011.

FOUCAULT, Michel. Vigilar y castigar. Madrid: S. XXI, 2000.

GAINTY, Denis. Martial Arts and the Body Politic in Meiji Japan. New York: Routledge, 2013.

GLEESON, Geoffrey. All About Judo. London: A. \& C. Black, 1984.

GUTTMANN, Allen; THOMPSON, Lee Austin. Japanese sports: a history. Honolulu: University of Hawaii, 2001.

HOBSBAWM, Eric. Introducción: La invención de la tradición. In: HOBSBAWM, E.; RANGER, T. La invención de la tradición. Barcelona: Editorial Crítica, 2002. p. 7-48.

IKEMOTO, Taku. Moral Education in Japan: implications for American schools. Disponible en: $<$ <ttp://www.hi-ho.ne.jp/taku77/papers/thes595.htm>. 1996. Acceso en: 5 ene. 2016.

INOUE, Sun. The invention of the martial arts: Kano Jigoro and Kodokan Judo. In: VLASTOS, S. Mirror of modernity: Invented traditions of modern Japan. Berkeley: University of California, 1998. p. 163-173.

KANO, Jigoro. Fondamenti del Judo. Milano: Luni Editrice, 1998.

KURISU, Mitsuru. A reexamination of physical education and sports in Japan (IV): Physical education and sports during the establishment of modern state. 2000. Disponible en: $<$ http://www. kyoto-seika.ac.jp/researchlab/wp/wp-content/uploads/kiyo/pdf-data/sa21/kurisu.pdf>. Acceso en: 25 ene. 2015.

LAVELLE, Pierre. La pensée politique du Japon contemporain (1868-1989). Paris: PUF, 1990.

MANZENREITER, Wolfram. Sport et politique du corps dans le Japon totalitaire. In: TSCHUDIN, J.-J.; HAMON, C. La société japonaise devant la montée du militarisme: Culture populaire et contrôle social dans les années 1930. Arles: Éditions Picquier, 2007. p. 71-90.

MAUSS, Marcel. Les techniques du corps. Communication présentée à la Société de Psychologie le 17 maio 1934. Article originalement publié Journal dePsychologie, v.32, n. 3-4, 1936. Disponible en: <http://classiques.uqac.ca/classiques/mauss marcel/socio et anthropo/6 Techniques corps/ Techniques corps.html> Acceso en : 27 sep. 2016.

MAZAC, M. Jigoro Kano: Père du judo. La vie du fondateur du judo. Noisy-Sur-École: Budo Editions, 2006.

NAGAI, Michio. El "despegue" y el "fracaso" en el desarrollo de la educación japonesa. Estudios Orientales, v. 4, n. 3, p. 247-279, 1969. 
NAKAJIMA, Tetsuya; THOMPSON, Lee. Judo and the process of nation-building in Japan: Kanō Jigorō and the formation of Kōdōkan judo. Asia Pacific Journal of Sport and Social Science, v. 1, n. 2-3, p. 97-110, 2012.

NARROWAY, Lisa. Symbols of State Ideology: the Samurai in Modern Japan. New Voices Volume, $n$. 2, p. 63-79, 2008.

NIEVES, Frank Stephen. The Modern Samurai: Martial Studies \& the Modernization of the Japanese School System. Thesis (Master of Culture Studies and Oriental Languages). 2014. Oslo: University of Oslo, 2014.

NIEHAUS, Andreas. If You Want to Cry, Cry on the Green Mats of Kodokan: Expressions of Japanese Cultural and National Identity in the Movement to Include Judo into the Olympic Programme. The International Journal of the History of Sport, v. 23, n. 7, p. 1173-1192, 2006.

PATTERSON, William R. Bushido's Role in the Growth of Pre-World War II Japanese Nationalism. Journal of Asian Martial Arts, v. 17, n. 3, p. 8-21, 2008.

ROBERTS, Jonathan. The gentle way to docility, an analysis of the implication and historical roots of the 1931 inclusion of judo in the Japanese middle school curriculum. 2009. Thesis (Master of East Asian Studies). - Toronto: University of Toronto, 2009.

SHIMAZU, Naoko. Nationalisms in Japan. Londres: Routledge, 2006.

SHIMIZU, Satoshi. The Body envisioned by Jigorō Kanō: Based on his career as a jūdo master, IOC member and principal of Tokyo Higher Normal School. Ido-Movement for Culture, v. 8, n. 8, p. 29-37, 2008.

STEVENS, John. Three budo masters. Tokyo: Kodansha International, 1995.

STEVENS, John. The Way of Judo: a Portrait of Jigoro Kano \& his Students. Boston: Shambhala, 2013.

UOZUMI, Takashi. An Outline of Budo History. In: UOZUMI, T. The History and Spirit of Budo. Katsuura: International Budo, 2010. p. 3-21.

WARNER, Gordon; DRAEGER, Don F. Japanese swordsmanship: Technique and practice. New York: Weatherhill, 1982.

WATSON, Brian. The Father of Judo: A Biography of Jigoro Kano. Tokio: Kodansha Internacional, 2000. 